\title{
Stochastic Finite Element Method for Torso Conductivity Uncertainties Quantification in Electrocardiography Inverse Problem
}

\author{
R. Aboulaich ${ }^{1 *}$, N. Fikal ${ }^{1}$, E. El Guarmah ${ }^{1,2}$, N. Zemzemi ${ }^{3,4}$ \\ ${ }^{1}$ Mohammed V University of Rabat, Mohammadia school of Engineering LERMA and LIRIMA \\ Laboratories. Av. Ibn Sina Agdal, Rabat Morocco \\ ${ }^{2}$ Royal Air School, Informatics and Mathematics Department DFST, BEFRA, POB40002, \\ Marrakech, Morocco
}

${ }^{3}$ INRIA Bordeaux Sud-Ouest, Carmen project 200 rue de la vieille tour 33405 Talence Cedex, France

${ }^{4}$ IHU Liryc, Electrophysiology and heart modeling institute. Avenue du Haut-Lévêque, 33604 Pessac, France

\begin{abstract}
The purpose of this paper is to study the influence of errors and uncertainties of the input data, like the conductivity, on the electrocardiography imaging (ECGI) solution. In order to do that, we propose a new stochastic optimal control formulation, permitting to calculate the distribution of the electric potentiel on the heart from the measurement on the body surface. The discretization is done using stochastic Galerkin method allowing to separate random and deterministic variables. Then, the problem is discretized, in spatial part, using the finite element method and the polynomial chaos expansion in the stochastic part of the problem. The considered problem is solved using a conjugate gradient method where the gradient of the cost function is computed with an adjoint technique. The efficiency of this approach to solve the inverse problem and the usability to quantify the effect of conductivity uncertainties in the torso are demonstrated through a number of numerical simulations on a 2D analytical geometry and on a $2 \mathrm{D}$ cross section of a real torso.
\end{abstract}

Keywords and phrases: electrocardiography forward problem, electrocardiography inverse problem, stochastic finite elements, chaos polynomial, uncertainty quantification, stochastic processes, stochastic Galerkin method

Mathematics Subject Classification: 35Q53, 34B20, 35G31

\section{Introduction}

In the last decade mathematical modeling in medicine and biological science, has shown important evolution. The research community has focused on the mathematical models for long time aiming to create more realistic models. Generally, models are imperfect abstractions or conception of reality. Moreover the value and utility of any model depends on the reliability and exactness of its input data, which are

\footnotetext{
*Corresponding author. E-mail: aboulaich@gmail.com
} 
rarely if ever available. On the other hand interaction between Input data errors and modeling uncertainties, leads to imprecision and uncertainty in model output. In order to improve the modelization and the simulation results, deterministic partial differential equation (PDE) have been reformulated into stochastic PDE (SPDE).

The ECGI problem consists of a forward problem and an inverse problem. For a given source current in the heart the forward problem, which is considered well posed, simulates the field distribution on the body surface by calculating the lead field. The inverse problem involves reconstruction of the primary source currents by localizing the electrical activity in the body using a set of measurement on the body surface and information about the torso geometry of the patient [17]. In this paper we consider the electrocardiographic forward and inverse problem . The electrical potential recovered on the heart surface allows to identify and localize some electrical dysfunctions. In the clinic inverse solutions, the goal is to target some triggers of cardiac arrhythmia and thereby plan a much more accurate surgical interventions $[24]$.

The mathematical inverse problem is known to be ill posed since the solution is generally not unique and is not stable [18]. Therefore the same problem can be formulated as minimization of an objective functional subject to PDE equation constraint, in our case it's a stochastic partial differential equation (SPDE). Many regularization methods have been developed in order to solve the obtained problem $[8,17,20,28]$. The obtained solution depends on the regularization method and parameters [19]. Although in the case of ECGI the inverse solution depends also on the physical parameters and the geometry of the patient. In most of the studies these variabilities have not been considered. In particular, the torso is assumed in the literature, in most of the studies to be homogenous. Moreover, when the conductivity heterogeneities are included, they are determined from data obtained from textbooks. The problem is that the difference between the experiment environments and other factors related to the measurement tools make this data to be different from a paper to another $[13,14]$.

Only few works have evaluated the effect of conductivities uncertainty in the propagation of the electrical potential in the torso [22,25]. Regarding the forward problem, authors in [15] use the stochastic finite elements method (SFEM) to describe the effect of lungs muscles and fat conductivities. In [25], a principal component approach has been used to predict the effect of conductivities variation on the body surface potential. However, to the best of our knowledge, no work in the literature has treated the influence of conductivity uncertainties of the ECGI inverse problem. In this work, we propose to use a stochastic optimal control approach to solve the inverse problem and to compute the potential value on the heart. Control cost functional will be formulated in terms of norms that include both spatial and stochastic dimensions. The derivation of the optimality system is analogous to the deterministic case in which one an energy functional has been used [1], with the SPDE constraint, as proposed in [15]. Moreover for the development of the optimization algorithm we use an iterative procedure based on the conjugate gradient method like in [1]. Then we take advantage of the fact that the expectation of the smooth random processes can be evaluated very conveniently with the stochastic galerkin (SG) method. In order to solve the SPDE problem, we use the stochastic finite element method. Full details about the SFEM could be found in $[3-5,16]$. However, few works are devoted to solving optimal control problems under uncertainty on the parameters of the models, and about the theoretical study we can see $[7,20]$. This paper is presented as follows:

In Section 1 we represent stochastic forward problem of electrocardiography, and we describe the application of the generalized polynomial chaos-stochastic Galerkin (gPC-SG) technique to solve the standard ECG forward problem.

In Section 2 we represent the ECGI inverse problem formulated as control problem involving an elliptic stochastic PDE constraint. We use an energy cost function and we calculate its gradient. We also provide the different steps of the conjugate gradient optimization algorithm.

In Section 3 we conduct 2D numerical simulation for the forward and inverse problems in an analytical case.

In Section 4 we perform numerical simulations on a 2D section of real life geometry of a torso. 
In Section 5 we analyze our obtained results. Finally, some conclusions are drawn in In Section 6

\section{Stochastic forward problem of electrocardiography}

\subsection{Function spaces and notation}

We give in the following a short overview of the notations, and definition of the stochastic Sobolev space used throughout this paper. Let $D$ be the spatial domain. $\Omega$ is sample space that belongs to a probability space $(\Omega, A, P), A$ denotes the $\sigma$-algebra of subsets of $\Omega$, and let $P$ be the probability measure. Following the theory of Wiener [26], as well as Xiu and Karniadakis [27], we can represent any general second-order random process $X(\omega), \omega \in \Omega$, in terms of a collection of finite number of random variables. We represent this random process by a vector $\boldsymbol{\xi}=\boldsymbol{\xi}(\omega)=\left(\xi_{1}(\omega), \ldots, \xi_{N}(\omega)\right) \in \mathbb{R}^{N}$, where $N$ is the dimension of the approximated stochastic space. We assume that each random variable is independent, its image space is given by $\Gamma_{i} \equiv \xi_{i}(\Omega) \subset \mathbb{R}$. Each random variable is characterised by a probability density function (PDF) $\rho_{i}: \Gamma_{i} \longrightarrow \mathbb{R}^{+}$, for $i=1, \ldots, N$. Then, we define the joint PDF of the random vector $\boldsymbol{\xi}$

$$
\rho(\boldsymbol{\xi})=\prod_{N}^{i=1} \rho_{i}\left(\xi_{i}\right) \quad \forall \boldsymbol{\xi} \in \Gamma
$$

where the support of $\rho$ is $\Gamma=\prod_{i=1}^{N} \Gamma_{i}$. The probability measure on $\Gamma$ is $\rho(\boldsymbol{\xi}) d \boldsymbol{\xi}$. As commented in [27], this allows us to conduct numerical formulations in the finite dimensional (N-dimensional) random space $\Gamma$. Let us denote $L^{2}(\Gamma)$ the space of random variables $X$ with finite second moments:

$$
\mathbb{E}\left[X^{2}(\boldsymbol{\xi})\right]=\int_{\Gamma} X^{2}(\boldsymbol{\xi}) \rho(\boldsymbol{\xi}) d \boldsymbol{\xi}<+\infty
$$

where $\mathbb{E}[$.$] denotes the mathematical expectation operator. This space is a Hilbert space with respect to$ the inner product:

$$
\langle X, Y\rangle_{L^{2}}=\mathbb{E}[X Y]=\int_{\Gamma}(X . Y) \rho(\boldsymbol{\xi}) d \boldsymbol{\xi} \quad \text { for } \mathrm{X}, \mathrm{Y} \in L^{2}(\Gamma) .
$$

Additionally, we consider a spatial domain $D$ and we define the tensor product Hilbert space $L^{2}(D) \otimes L^{2}(\Gamma)$ of second-order random fields as:

$$
L^{2}(D) \otimes L^{2}(\Gamma)=\left\{u: D \otimes \Omega \longrightarrow \mathbb{R}, \int_{\Omega} \int_{D}|u(x, \boldsymbol{\xi})|^{2} d x \rho(\boldsymbol{\xi}) d \boldsymbol{\xi}<\infty\right\} .
$$

This space is equipped with the norm:

$$
\|u\|_{L^{2}(D) \otimes L^{2}(\Gamma)}=\left(\left(\int_{\Omega} \int_{D}|u|^{2} d x \rho(\boldsymbol{\xi}) d \boldsymbol{\xi}\right)\right)^{\frac{1}{2}} .
$$

Analogously, the tensor product spaces $H^{1}(D) \otimes L^{2}(\Gamma)$ and $H_{0}^{1}(D) \otimes L^{2}(\Gamma)$ can be defined.

\subsection{Stochastic formulation of the forward problem}

Following [15] we represent the stochastic characteristics of the forward solution of the Laplace equation by the generalized chaos polynomial. For the space domain we use simplified analytical 2D model representing a cross-section of the torso (see Figure 1) in which the conductivities vary stochastically.

Since we suppose that the conductivity parameter depends on the space and on the stochastic variable $\sigma(x, \xi)$, the solution of the Laplace equation does also depends on space and the stochastic variable $u(x, \xi))$. The stochastic forward problem of electrocardiography can be written as follows

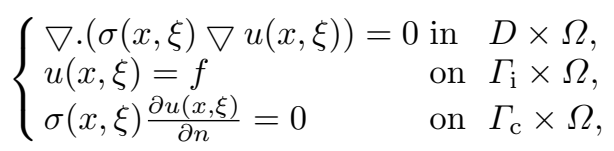

where, $\Gamma_{\mathrm{i}}$ and $\Gamma_{\mathrm{c}}$ are the epicardial and torso boundaries respectively, $f$ is the potential at the epicardial boundary and $\xi \in \Omega$ is the stochastic variable. Without lost of generality the stochastic variable could 


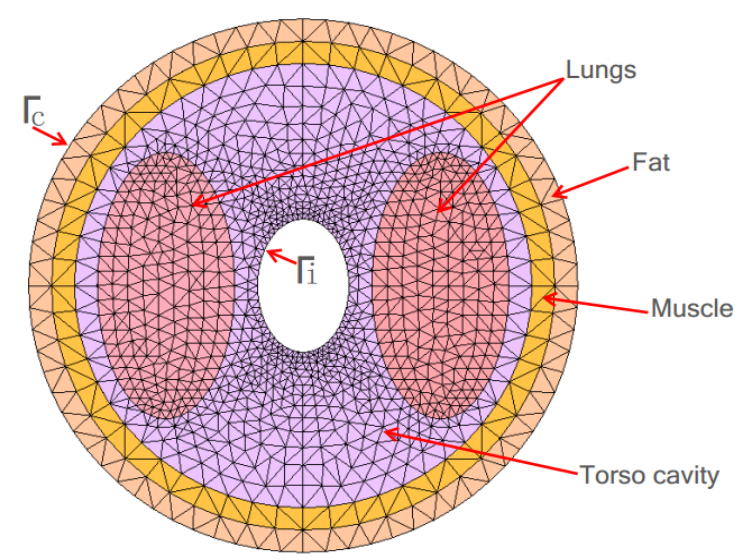

Figure 1: 2D computational mesh of the torso geometry showing the different regions of the torso considered in this study (fat, muscle, lungs, torso cavity)

also be represented by a vector. The numerical results in [15] show that there is no difference between using one ore second dimensional stochastic spaces. The notion of the weak solution for SPDEs is based on a extension of classical theory [4], test function become random fields and an integration over stochastic space is done with respect to the corresponding measure. Thus, the weak form involves expectations of the weak problem formulation in the physical space. Correspondingly, denoting by $u_{0}$ the extension of $f$ to the whole domain, and $\tilde{u} \in H_{0}^{1}(D) \otimes L^{2}(\Gamma), \tilde{u}=u-u_{0}$ is the weak solution of (2.1), if for all test functions $v \in H_{0}^{1}(D) \otimes L^{2}(\Gamma),[3],[10]$, we have

$$
\mathbb{E}\left[\int_{D} \sigma(x, \xi) \nabla \tilde{u}(x, \xi) \cdot \nabla v(x, \xi) d x\right]+\mathbb{E}\left[\int_{D} \sigma(x, \xi) \nabla u_{0}(x, \xi) \cdot \nabla v(x, \xi) d x\right]=0 .
$$

\subsection{Descretization of the stochastic forward problem}

In order to compute approximate solutions, we use the stochastic Galerkin (SG) method to solve equation (2.1). To develop this method, we denote $Y_{p} \subset L^{2}(\Gamma)$ the stochastic approximation space

$$
Y_{p}=\operatorname{span}\left\{\Psi_{0}, \ldots, \Psi_{p}\right\} \text {. }
$$

A stochastic process $X(\xi)$ of a parameter or a variable $X$ is represented by weighted sum of orthogonal polynomials $\left\{\Psi_{i}(\xi)\right\}$ denoting the generalized chaos polynomial. More details about the different choices of PDFs could be found in [21]. In our case we use the Legendre polynomials which are more suitable for uniform probability density. We have

$$
X(\xi)=\sum_{i=0}^{p} \hat{X}_{i} \Psi_{i}(\xi),
$$

where $\hat{X}_{i}$ are the projections of the random process on the stochastic basis $\left\{\Psi_{i}(\xi)\right\}_{i=1}^{p}$ with respect to the joint $\mathrm{PDF} \rho$.

$$
\hat{X}_{i}=\int_{\Omega} X(\xi) \Psi_{i}(\xi) d \rho=\left\langle X(\xi) \cdot \Psi_{i}(\xi)\right\rangle_{\rho} .
$$

The mean value and the standard deviation of $\mathrm{X}$ over $\Omega$ are then computed as follows

$$
\mathbb{E}(X)=\int_{\Omega} \sum_{i=0}^{p} \hat{X}_{i} \Psi_{i}(\xi)=\hat{X}_{0},
$$




$$
\operatorname{stdev}[X]=\left(\sum_{i=1}^{p} \hat{X}_{i}^{2} \int_{\Omega} \Psi_{i}(\xi)^{2}\right)^{\frac{1}{2}}
$$

Since in our study we would like to evaluate the effect of the conductivity randomness of the different torso organs on the electrical potential, both of $\sigma$ and $u$ are now expressed in the Galerkin space as follows:

$$
u(x, \xi)=\sum_{k=0}^{p} \hat{u}_{k}(x) \Psi_{k}(\xi) \quad \text { (a) } \quad \sigma(x, \xi)=\sum_{l=0}^{p} \hat{\sigma}_{l}(x) \Psi_{l}(\xi)
$$

By substituting (a), (b) into the elliptic equation (2.1) and by projecting the result on the polynomial basis $\left\{\Psi_{m}(\xi)\right\}_{m=1}^{p}$, we obtain the following system:

For $m=0, \ldots, p$,

$$
\begin{aligned}
\left.\sum_{l=0}^{p} \sum_{k=0}^{p} T_{k l m} \nabla \cdot\left(\hat{\sigma}_{l}(x) \nabla\right) \hat{u}_{k}(x)\right) & =0 & & \text { in } D, \\
\hat{u}_{0}(x) & =u_{0}(x) & & \text { on } \Gamma_{\mathrm{i}}, \\
\hat{u}_{k}(x) & =0 & & \text { on } \Gamma_{\mathrm{i}} \forall k=1, \ldots p, \\
\hat{\sigma}_{l}(x) \frac{\partial \hat{u}_{k}(x)}{\partial n} & =0 & & \text { on } \Gamma_{\mathrm{c}} \forall l, k=0, \ldots p,
\end{aligned}
$$

where $T_{k l m}=\mathbb{E}\left[\Psi_{k}(\xi), \Psi_{l}(\xi), \Psi_{m}(\xi)\right]$.

For the spatial domain, we define a subspace $V_{h} \subset H_{0}^{1}(D)$ of standard Lagrange finite element functions on a triangulation of the domain D.

$$
V_{h}:=\operatorname{span}\left\{\phi_{1}, \phi_{2}, \ldots, \phi_{N}\right\}
$$

By applying the standard finite elements variational formulation and Galerkin projections we obtain a linear system of size $(p \times N)$, where $N$ is the number of the degrees of freedom for the Laplace equation in the deterministic framework. Obviously $u \in Y_{p} \otimes V_{h}$ the electrical potential is now expressed in this tensor product subspaces as follows:

$$
u(x, \xi)=\sum_{k=0}^{p} \sum_{i=0}^{N}\left(u_{k}\right)_{i} \phi_{i} \Psi_{k} .
$$

The system (2.3) can be reformulated as linear combination of finite elements stiffeness matrices which results is

$$
\sum_{k=0}^{p}\left[T_{k l m} K^{l}\right] \hat{u}_{k}(x)=\sum_{k=0}^{p} T_{k l m} f^{l},
$$

where $\hat{u}_{k}(x)$ denotes the vector of finite element degrees of freedom expressing the $k^{\text {th }}$ stochastic mode, and for $l=1, \ldots, p$

$$
\begin{gathered}
K^{l}=\left[K^{l}\right]_{i j}=\left(\sigma_{l} \nabla \phi_{i} \cdot \nabla \phi_{j}\right), \\
f^{l}=\left(f_{j}^{l}\right)_{j}=\sum_{x_{i} \in \Gamma_{\mathrm{i}}}\left(\hat{u}_{k}\right)_{i}\left(\sigma_{l} \nabla \phi_{i} . \nabla \phi_{j}\right) .
\end{gathered}
$$

The symbol (.) denotes the inner product taken over the entire spatial domain.

\section{Stochastic inverse problem of electrocardiography}

The inverse problem in electrocadiography imaging (ECGI) is a technique that allows to construct the electrical potential on the heart surface $\Gamma_{\mathrm{i}}$ from data measured on the body surface $\Gamma_{\mathrm{c}}$. Taking into account the variability of the tissue conductivities in the torso, we assume that the electrical potential 
is governed by the stochastic diffusion equation as shown in the previous section. For a given potential data $f$ on the body surface $\Gamma_{\mathrm{c}}$, the goal is to find $u(x, \xi)$ on $\Gamma_{\mathrm{i}}$ such that the potential data in the torso domain satisfies

$$
\begin{cases}\nabla \cdot(\sigma(x, \xi) \nabla u(x, \xi))=0 & \text { in } D \times \Omega \\ u(x, \xi)=f & \text { on } \Gamma_{\mathrm{c}} \times \Omega \\ \sigma(x, \xi) \frac{\partial u(x, \xi)}{\partial n}=0 & \text { on } \Gamma_{\mathrm{c}} \times \Omega .\end{cases}
$$

Mathematically (3.1) represent a data completion Cauchy problem for the stochastic diffusion equation. This problem is known to be ill-posed since Hadamard[18]. In order to reconstruct the lacking data $u(x, \xi)$ on $\Gamma_{\mathrm{i}} \times \Omega$, we propose in this paper to build an optimal control problem that takes into account the uncertainties in the torso conductivities. We then use an energy cost function as described in $[1,2]$ constrained by the stochastic diffusion equation. In order to generate compatible Cauchy data, we solve a deterministic forward problem. We denote by $u_{T}$ the forward solution. Then, we extract the electrical potential at the external boundary and we denote it by $f=u_{T / \Gamma_{\mathrm{c}}}$.

We look for $(\eta, \tau) \in L^{2}\left(\Gamma_{\mathrm{i}}\right) \times L^{2}\left(\Gamma_{\mathrm{i}}\right)$ minimizing the following cost function with a least square setting, where the Dirichlet boundary condition is used as data, the other boundary condition (normal derivative) is used as a control, meaning that at each iteration only one forward problem will be solved, unlike [1] which in the authors have used a splitting approach. In (3.1) We suppose that the flux on the external boundary is nul but we look for the internal flux which is computed as a control term $\eta$ in the problem (3.2). Moreover for the regularity of boundary data, like in [1] we suppose that $\partial D$ is $C^{2}$ and has two connected components $\Gamma_{\mathrm{c}}$ and $\Gamma_{\mathrm{i}}, f \in H^{\frac{1}{2}}\left(\Gamma_{\mathrm{c}}\right)$, we can then suppose that $u(x, \xi) \in H^{\frac{3}{2}}(D) \otimes L^{2}(\Gamma)$ and $\sigma(x, \xi) \frac{\partial u(x, \xi)}{\partial n} \in L^{2}\left(\Gamma_{\mathrm{i}}\right) \otimes L^{2}(\Gamma)$.

$$
\begin{cases}J(\eta, \tau)=\frac{1}{2} \mathbb{E}\left(\int_{\Gamma_{\mathrm{c}}}(v(x, \xi)-f)^{2} d \Gamma_{\mathrm{c}}+\int_{\Gamma_{\mathrm{i}}}\left(\sigma(x, \xi) \frac{\partial v(x, \xi)}{\partial n}-\eta\right)^{2} d \Gamma_{\mathrm{i}}\right), \\ \text { with } v(x, \xi) \text { solution of : } & \text { in } D \times \Omega \\ \nabla \cdot(\sigma(x, \xi) \nabla v(x, \xi))=0 & \text { on } \Gamma_{\mathrm{i}} \times \Omega \\ v(x, \xi)=\tau & \text { on } \Gamma_{\mathrm{c}} \times \Omega . \\ \sigma(x, \xi) \frac{\partial v(x, \xi)}{\partial n}=0 & \text { in }\end{cases}
$$

The differentiability of $J$ and the equivalence of this minimization problem with the completion one (3.1), results are similar to the determistic case $[1,23]$. In order to solve this minimization problem, we use a conjugate gradient method as introduced for solving the data completion problem in the deterministic case [1]. In this work, the components of the gradient of the cost function are computed using an adjoint method. The derivation of the optimality system 3.2 is described in the following paragraph.

Remark 3.1. We suppose in the following that the Dirichlet data are in $H^{\frac{1}{2}}\left(\Gamma_{\mathrm{c}}\right)$ and the Neumann data are in $L^{2}\left(\Gamma_{\mathrm{i}}\right)$ and we consider the space $W=\left\{v \in H^{1}(D) / v \mid \Gamma_{\mathrm{i}}=0\right\}$ and we denote by $\mathcal{W}=W \otimes L^{2}(\Gamma)$.

\subsection{Computation of the gradients}

Lemma 3.2. The gradient of the cost function $J$ with respect to $\eta$ and $\tau$ is given by:

$$
\begin{cases}\frac{\partial J(\eta, \tau)}{\partial \eta} \cdot \vartheta=-\mathbb{E}\left[\int_{\Gamma_{i}}\left(\sigma \frac{\partial v}{\partial n}-\eta\right) \vartheta d \Gamma_{i}\right] & \forall \vartheta \in L^{2}\left(\Gamma_{i}\right) \\ \frac{\partial J(\eta, \tau)}{\partial \tau} \cdot h=\mathbb{E}\left[\int_{\Gamma_{i}} \sigma \frac{\partial \lambda}{\partial n} h d \Gamma_{i}\right] & \forall h \in L^{2}\left(\Gamma_{i}\right) \\ \text { with } \lambda \text { solution of: } & \\ \nabla \cdot(\sigma(x, \xi) \nabla \lambda(x, \xi))=0 & \text { in } D \times \Omega \\ \lambda(x, \xi)=\sigma(x, \xi) \frac{\partial v(x, \xi)}{\partial n}-\eta & \text { on } \Gamma_{i} \times \Omega \\ \sigma(x, \xi) \frac{\partial \lambda(x, \xi)}{\partial n}=-(v-f) & \text { on } \Gamma_{c} \times \Omega\end{cases}
$$


Proof. First, we compute the derivative of the cost function with respect to the variable $\eta$. Since the function $v$ is independent of $\eta$, we obtain:

$$
\frac{\partial J(\eta, \tau)}{\partial \eta} \cdot \vartheta=-\mathbb{E}\left[\int_{\Gamma_{\mathrm{i}}}\left(\sigma \frac{\partial v}{\partial n}-\eta\right) \vartheta d \Gamma_{\mathrm{i}}\right] \quad \forall \vartheta \in L^{2}\left(\Gamma_{\mathrm{i}}\right) .
$$

Analogously deriving $J$ with respect to the second variable gives:

$$
\frac{\partial J(\eta, \tau)}{\partial \tau} \cdot h=\mathbb{E}\left[\int_{\Gamma_{\mathrm{c}}}(v-f) v^{\prime}(h) d \Gamma^{c}\right]+\mathbb{E}\left[\int_{\Gamma_{\mathrm{i}}}\left(\sigma \frac{\partial v}{\partial n}-\eta\right) \sigma \frac{\partial v^{\prime}(h)}{\partial n} d \Gamma_{\mathrm{i}}\right] \quad \forall h \in L^{2}\left(\Gamma_{\mathrm{i}}\right),
$$

where $v^{\prime}(h)=\frac{\partial v}{\partial \tau}(h)$. The expression (3.5) could not be used in practice, mainly, because we cannot deduce $\frac{\partial J(\eta, \tau)}{\partial \tau}$ from it. In order to calculate this gradient, we use an adjoint method.

The Lagrange function is defined as follows:

$$
\mathcal{L}(\eta, \tau, v, \lambda)=\frac{1}{2} \mathbb{E}\left[\int_{\Gamma_{\mathrm{c}}}(v-f)^{2} d \Gamma_{\mathrm{c}}\right]+\frac{1}{2} \mathbb{E}\left[\int_{\Gamma_{\mathrm{i}}}\left(\sigma \frac{\partial v}{\partial n}-\eta\right)^{2} d \Gamma_{\mathrm{i}}\right]+\mathbb{E}\left[\int_{D} \sigma \nabla v \nabla \lambda d D-\int_{\Gamma_{\mathrm{i}}} \sigma \frac{\partial v}{\partial n} \lambda d \Gamma_{\mathrm{i}}\right]
$$

The random field $\lambda(x, \xi) \in H^{\frac{3}{2}}(D) \otimes L^{2}(\Gamma)$ is the Lagrange multiplier of the SPDE constraint of (3.2), but for the computation we use the norm space of $H^{1}(D) \otimes L^{2}(\Gamma)$. Its equation is obtained by deriving the Lagrange equation with respect to $v$ :

$$
\frac{\partial \mathcal{L}}{\partial v}(\eta, \tau, v, \lambda) \cdot \phi=\mathbb{E}\left[\int_{\Gamma_{\mathrm{c}}}(v-f) \phi d \Gamma_{\mathrm{c}}\right]+\mathbb{E}\left[\int_{\Gamma_{\mathrm{i}}}\left(\sigma \frac{\partial v}{\partial n}-\eta\right) \sigma \frac{\partial \phi}{\partial n} d \Gamma_{\mathrm{i}}\right]+\mathbb{E}\left[\int_{D} \sigma \nabla \phi \nabla \lambda d D-\int_{\Gamma_{\mathrm{i}}} \sigma \frac{\partial \phi}{\partial n} \lambda d \Gamma_{\mathrm{i}}\right] .
$$

Then for $\frac{\partial L}{\partial v}(\eta, \tau, v, \lambda) . \phi=0$, with $\phi \in \mathcal{W}$, we get :

$$
\mathbb{E}\left[\int_{D} \sigma \nabla \phi \nabla \lambda d D\right]=\mathbb{E}\left[\int_{\Gamma_{\mathrm{i}}} \sigma \frac{\partial \phi}{\partial n} \lambda d \Gamma_{\mathrm{i}}\right]-\mathbb{E}\left[\int_{\Gamma_{\mathrm{c}}}(v-f) \phi d \Gamma_{\mathrm{c}}\right]-\mathbb{E}\left[\int_{\Gamma_{\mathrm{i}}}\left(\sigma \frac{\partial v}{\partial n}-\eta\right) \sigma \frac{\partial \phi}{\partial n} d \Gamma_{\mathrm{i}}\right]
$$

By applying Green's formula in (3.6) we obtain:

$$
-\mathbb{E}\left[\int_{D} \nabla \cdot(\sigma \nabla \lambda) \phi d D\right]+\mathbb{E}\left[\int_{\Gamma_{\mathrm{i}}} \sigma \frac{\partial \lambda}{\partial n} \phi d \Gamma_{\mathrm{i}}\right]+\mathbb{E}\left[\int_{\Gamma_{\mathrm{c}}} \sigma \frac{\partial \lambda}{\partial n} \phi d \Gamma_{\mathrm{c}}\right]=\mathbb{E}\left[\int_{D} \sigma \nabla \lambda \nabla \phi d D\right] .
$$

Since $\phi \in \mathcal{W}$, we have $\phi=0$ on $\Gamma_{\mathrm{i}}$, the equation (3.7) becomes:

$$
-\mathbb{E}\left[\int_{D} \nabla \cdot(\sigma \nabla \lambda) \phi d D\right]+\mathbb{E}\left[\int_{\Gamma_{\mathrm{c}}} \sigma \frac{\partial \lambda}{\partial n} \phi d \Gamma_{\mathrm{c}}\right]=\mathbb{E}\left[\int_{D} \sigma \nabla \lambda \nabla \phi d D\right] .
$$

Combining (3.6) and (3.8) we finally get:

$$
-\mathbb{E}\left[\int_{D} \nabla \cdot(\sigma \nabla \lambda) \phi d D\right]+\mathbb{E}\left[\int_{\Gamma_{\mathrm{c}}} \sigma \frac{\partial \lambda}{\partial n} \phi d \Gamma_{\mathrm{c}}\right]=\mathbb{E}\left[\int_{\Gamma_{\mathrm{i}}} \sigma \frac{\partial \phi}{\partial n}\left[\lambda-\left(\sigma \frac{\partial v}{\partial n}-\eta\right)\right] d \Gamma_{\mathrm{i}}\right]-\mathbb{E}\left[\int_{\Gamma_{\mathrm{c}}}(v-f) \phi d \Gamma_{\mathrm{c}}\right] .
$$

Then, by gathering the adequate terms we obtain:

$$
\mathbb{E}\left[\int_{D} \nabla \cdot(\sigma \nabla \lambda) \phi d D\right]=-\mathbb{E}\left[\int_{\Gamma_{\mathrm{i}}} \sigma \frac{\partial \phi}{\partial n}\left[\lambda-\left(\sigma \frac{\partial v}{\partial n}-\eta\right)\right] d \Gamma_{\mathrm{i}}\right]+\mathbb{E}\left[\int_{\Gamma_{\mathrm{c}}}\left(v-f+\sigma \frac{\partial \lambda}{\partial n}\right) \phi d \Gamma_{\mathrm{c}}\right] .
$$

Finally, we deduce that the solution of the following adjoint system is also solution of the variational system (3.10),

$$
\left\{\begin{array}{ccc}
\nabla \cdot(\sigma \nabla \lambda)=0 & \text { in } & D \times \Omega \\
\lambda=\sigma \frac{\partial v}{\partial n}-\eta & \text { on } & \Gamma_{\mathrm{i}} \times \Omega \\
\sigma \frac{\partial \lambda}{\partial n}=-(v-f) & \text { on } & \Gamma_{\mathrm{c}} \times \Omega
\end{array}\right.
$$


The corresponding variational problem is :

$$
\mathbb{E}\left[\int_{D} \sigma \nabla \lambda \nabla \phi d D\right]=\mathbb{E}\left[\int_{\Gamma_{\mathrm{i}}} \sigma \frac{\partial \lambda}{\partial n} \phi d \Gamma_{\mathrm{i}}\right]-\mathbb{E}\left[\int_{\Gamma_{\mathrm{c}}}(v-f) \phi d \Gamma_{\mathrm{c}}\right] .
$$

By replacing $\phi$ with $v^{\prime}$ in $(3.12)$, we obtain:

$$
\mathbb{E}\left[\int_{D} \sigma \nabla \lambda \nabla v^{\prime} d D\right]=\mathbb{E}\left[\int_{\Gamma_{\mathrm{i}}} \sigma \frac{\partial \lambda}{\partial n} v^{\prime} d \Gamma_{\mathrm{i}}\right]-\mathbb{E}\left[\int_{\Gamma_{\mathrm{c}}}(v-f) v^{\prime} d \Gamma_{\mathrm{c}}\right] .
$$

On the other hand, deriving the state problem (3.1) with respect to $\tau$ gives the following equation

$$
\left\{\begin{array}{llr}
\nabla \cdot\left(\sigma \nabla v^{\prime}\right)=0 & \text { in } & D \times \Omega \\
\sigma \frac{\partial v^{\prime}}{\partial n}=0 & \text { on } & \Gamma_{\mathrm{c}} \times \Omega \\
v^{\prime}=h & \text { on } & \Gamma_{\mathrm{i}} \times \Omega
\end{array}\right.
$$

The variational formulation of this problem is:

$$
\mathbb{E}\left[\int_{D} \sigma \nabla v^{\prime} \nabla \omega d D\right]=\mathbb{E}\left[\int_{\Gamma_{\mathrm{i}}} \sigma \frac{\partial v^{\prime}}{\partial n} \omega d \Gamma_{\mathrm{i}}\right] \quad \forall \omega \in H^{1}(D) \otimes L^{2}(\Gamma) .
$$

Assuming that $\omega=\lambda$ we have:

$$
\mathbb{E}\left[\int_{D} \sigma \nabla v^{\prime} \nabla \lambda d D\right]=\mathbb{E}\left[\int_{\Gamma_{\mathrm{i}}} \sigma \frac{\partial v^{\prime}}{\partial n} \lambda d \Gamma_{\mathrm{i}}\right]
$$

hence

$$
\mathbb{E}\left[\int_{\Gamma_{\mathrm{i}}} \sigma \frac{\partial v^{\prime}}{\partial n} \lambda d \Gamma_{\mathrm{i}}\right]=\mathbb{E}\left[\int_{\Gamma_{\mathrm{i}}} \sigma \frac{\partial \lambda}{\partial n} v^{\prime} d \Gamma_{\mathrm{i}}\right]-\mathbb{E}\left[\int_{\Gamma_{\mathrm{c}}}(v-f) v^{\prime} d \Gamma_{\mathrm{c}}\right]
$$

Then, we obtain

$$
\mathbb{E}\left[\int_{\Gamma_{\mathrm{c}}}(v-f) v^{\prime} d \Gamma_{\mathrm{c}}\right]=\mathbb{E}\left[\int_{\Gamma_{\mathrm{i}}} \sigma \frac{\partial \lambda}{\partial n} v^{\prime} d \Gamma_{\mathrm{i}}\right]-\mathbb{E}\left[\int_{\Gamma_{\mathrm{i}}} \sigma \frac{\partial v^{\prime}}{\partial n} \lambda d \Gamma_{\mathrm{i}}\right] .
$$

Using (3.5) and since $\lambda=\left(\sigma \frac{\partial v}{\partial n}-\eta\right) \mid \Gamma_{\mathrm{i}}$ we get,

$$
\frac{\partial J(\eta, \tau)}{\partial \tau} \cdot h=\mathbb{E}\left[\int_{\Gamma_{\mathrm{c}}}(v-f) v^{\prime}(h) d \Gamma^{c}\right]+\mathbb{E}\left[\int_{\Gamma_{\mathrm{i}}} \lambda \sigma \frac{\partial v^{\prime}}{\partial n} d \Gamma_{\mathrm{i}}\right] .
$$

From the equations (3.14), (3.18) and (3.19) we finally obtain:

$$
\frac{\partial J(\eta, \tau)}{\partial \tau} \cdot h=\mathbb{E}\left[\int_{\Gamma_{\mathrm{i}}} \sigma \frac{\partial \lambda}{\partial n} h d \Gamma_{\mathrm{i}}\right] .
$$

\subsection{The conjugate gradient algorithm.}

In the previous section, we reformulated the stochastic Cauchy problem as a minimization problem. In order to numerically solve this problem, we use a conjugate gradient optimization procedure. The different steps of the algorithm are performed as follows:

Step 1. Given $f \in L^{2}\left(\Gamma_{\mathrm{c}}\right)$ choose an arbitrary initial guess

$$
\left(\varphi_{p}, t_{p}\right) \in L^{2}\left(\Gamma_{\mathrm{i}}\right) \times L^{2}\left(\Gamma_{\mathrm{i}}\right) .
$$


Step 1.1. Solve the well-posed stochastic forward problem:

$$
\begin{cases}\nabla \cdot\left(\sigma(x, \xi) \nabla v^{p}(x, \xi)\right)=0 & \text { in } D \times \Omega \\ \sigma(x, \xi) \frac{\partial v^{p}(x, \xi)}{\partial n}=0 & \text { on } \Gamma_{\mathrm{c}} \times \Omega \\ v^{p}(x, \xi)=t_{p} & \text { on } \Gamma_{\mathrm{i}} \times \Omega\end{cases}
$$

in order to obtain $v^{p} \mid \Gamma_{\mathrm{i}}$ and $\sigma \frac{\partial v^{p}}{\partial n} \mid \Gamma_{\mathrm{i}}$.

Step 1.2. Solve the stochastic adjoint problem:

$$
\left\{\begin{array}{lll}
\nabla \cdot\left(\sigma(x, \xi) \nabla \lambda^{p}(x, \xi)\right)=0 & \text { in } & D \times \Omega \\
\lambda^{p}(x, \xi)=\sigma(x, \xi) \frac{\partial v^{p}(x, \xi)}{\partial n}-\varphi_{p} & \text { on } & \Gamma_{\mathrm{i}} \times \Omega \\
\sigma(x, \xi) \frac{\partial \lambda^{p}(x, \xi)}{\partial n}=-\left(v^{p}(x, \xi)-f\right) & \text { on } & \Gamma_{\mathrm{c}} \times \Omega
\end{array}\right.
$$

in order to obtain $\lambda_{/ \Gamma_{\mathrm{i}}}^{p}$, and $\sigma \frac{\partial \lambda^{p}}{\partial n} / \Gamma_{\mathrm{i}}$.

step 1.3.: We evaluate the gradient:

$$
\nabla J\left(\varphi_{p}, t_{p}\right)=\left(\mathbb{E}\left[\varphi^{p}-\sigma(x, \xi) \frac{\partial v^{p}(x, \xi)}{\partial n}\right], \mathbb{E}\left[\sigma(x, \xi) \frac{\partial \lambda^{p}(x, \xi)}{\partial n}\right]\right) .
$$

Step 1.4. Determine the descent direction $d_{p}$ as follows:

$$
\left\{\begin{array}{l}
\gamma_{p-1}=\frac{\left\|\nabla J\left(\varphi_{p}, t_{p}\right)\right\|^{2}}{\left\|\nabla J\left(\varphi_{p-1}, t_{p}-1\right)\right\|^{2}} \\
d_{p}:=\left(d_{1}^{p}, d_{2}^{p}\right)=-\nabla J\left(\varphi_{p}, t_{p}\right)+\gamma_{p-1} d_{p-1}
\end{array}\right.
$$

in order to obtain:

$$
\left(\varphi_{p+1}, t_{p+1}\right)=\left(\varphi_{p}, t_{p}\right)+\alpha_{p} d_{p}
$$

where the scalar $\alpha_{p}$ is obtained through a linear search by:

$$
\alpha_{p}=-\frac{\mathbb{E}\left[\int_{\Gamma_{\mathrm{c}}} z^{p}\left(v^{p}-f\right) d \Gamma_{\mathrm{c}}\right]+\mathbb{E}\left[\int_{\Gamma_{\mathrm{i}}}\left(\sigma \frac{\partial z^{p}}{\partial n}-d_{1}^{p}\right)\left(\sigma \frac{\partial v^{p}}{\partial n}-\varphi_{p}\right) d \Gamma_{\mathrm{i}}\right]}{\mathbb{E}\left[\int_{\Gamma_{\mathrm{c}}}\left(z^{p}\right)^{2} d \Gamma_{\mathrm{c}}\right]+\mathbb{E}\left[\int_{\Gamma_{\mathrm{i}}}\left(\sigma \frac{\partial z^{p}}{\partial n}-d_{1}^{p}\right)^{2} d \Gamma_{\mathrm{i}}\right]} .
$$

We note that $z^{p}$ is the solution of:

$$
\left\{\begin{array}{lll}
\nabla \cdot\left(\sigma(x, \xi) \nabla z^{p}(x, \xi)\right)=0 & \text { in } & D \times \Omega \\
z^{p}(x, \xi)=d_{2}^{p} & \text { on } & \Gamma_{\mathrm{i}} \times \Omega \\
\sigma(x, \xi) \frac{\partial z^{p}(x, \xi)}{\partial n}=0 & \text { on } & \Gamma_{\mathrm{c}} \times \Omega
\end{array}\right.
$$

Step 2. Having obtained $\left(\varphi_{p}, t_{p}\right)$ for $p \geq 0$, set $p=p+1$ and repeat from step 1.1 until the prescribed stopping criterion is satisfied. For the stopping criterion, our algorithm stops when $J\left(\varphi_{p}, t_{p}\right) \leq \epsilon$ or when $\left\|\nabla J\left(\varphi_{p}, t_{p}\right)\right\|_{L^{2}\left(\Gamma_{\mathrm{i}}\right)} \leq \epsilon_{1}$, where $\epsilon$ is the objective function tolerence and $\epsilon_{1}$ is its gradient tolerence.

\section{Numerical results: Analytical case}

In this section we present the numerical results of the stochastic forward and inverse problems. In order to assess the effect of the conductivity uncertainties of each of organs conductivities on the electrical potential at the heart boundary, we start by generating our ground truth solution. For the sake of simplicity and reproducibility we take a harmonic function on the heart boundary, where the exact extracellular potential:

$$
u_{e x}(x, y)=\exp (x) \sin (y)
$$




\begin{tabular}{|c|c|c|}
\hline organ category & major radius $(\mathrm{cm})$ & minor radius $(\mathrm{cm})$ \\
\hline heart & 1.5 & 1 \\
lungs & 3.5 & 1.5 \\
torso cavity & 5 & 5 \\
muscle & 5.5 & 5.5 \\
fat & 6 & 6 \\
\hline
\end{tabular}

Table 1: Values of the minor and major radius of the ellipsoids representing organs regions.

The heart surface the lungs the muscle and fat domains are defined using ellipsoids geometries as shown in Figure 1. Values of minor and major radius of these ellipsoids are given in Table 1 for all organs. Since we assume that the uncertainty of the conductivity value follows a uniform probability density, as chaos polynomial basis $\left\{\Psi_{i}\right\}$ we use the Legendre polynomials defined on the interval $\Omega=[-1,1]$. We also suppose that the true conductivity uncertainty interval is centered in $\sigma_{T}$, which we obtain from the literature $[9,11]$. Table 2 summarizes the mean values of the conductivities.

\begin{tabular}{|c|c|}
\hline organ category & conductivity $(\mathrm{S} / \mathrm{m})$ \\
\hline lungs & 0.096 \\
muscle & 0.200 \\
fat & 0.045 \\
torso cavity & 0.239 \\
\hline
\end{tabular}

Table 2: Conductivity values corresponding to the organs that are considered in the model.

\subsection{Sensitivity of the forward problem to the conductivity uncertainties}

In order to isolate the effect of each of the torso organs conductivity uncertainties on the forward problem, we suppose that all of the organs conductivities are known (deterministic) except one. Then, we solve the stochastic forward problem (2.1). This test has been performed in [15]. In order to validate our forward problem, we perform this test for all organs conductivities.

In Figure 2 (a), we show the mean value of the stochastic forward solution. Due to the linearity of the problem, to the fact that we choose the uniform law and the fact that the center of the interval of the stochastic variable corresponds to the exact conductivity, the mean value of the stochastic solution is equal to the deterministic forward solution ( Figure 2 (b)). In Figure 2 (c), (respectively (d) , (e) and (f)), we show the standard deviation of the stochastic forward solution for $\pm 50 \%$ uncertainty on the fat (respectively, muscle, lungs and torso cavity) conductivity value. First, we see that the maximum values of the standard deviation are small compared to the mean value of the potential, it is $4 \%$ for the torso cavity, $2 \%$ for lungs, $10^{-4}$ for muscle and $10^{-5}$ for fat .

This means that the forward solution is more sensitive to the torso cavity and lung conductivities than it is for the muscle and fat. Second, one could remark that for all cases, the maximum value of the standard deviation is reached at the edge of the corresponding organ. In both cases the effect of the conductivity uncertainties on the forward solution does not exceed $4 \%$ of the value of the potential, which means that, relatively, the forward solution is slightly affected by the conductivity uncertainties. 


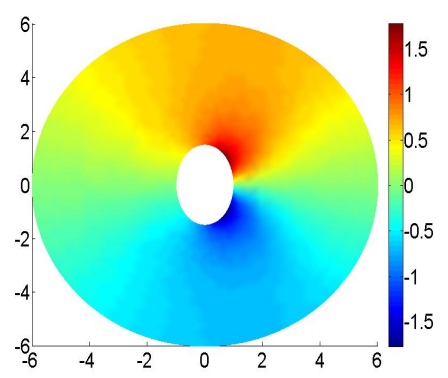

(a)

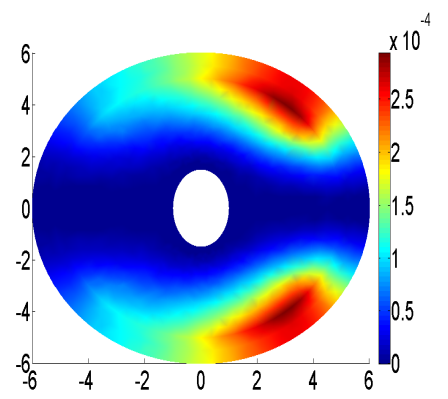

(d)

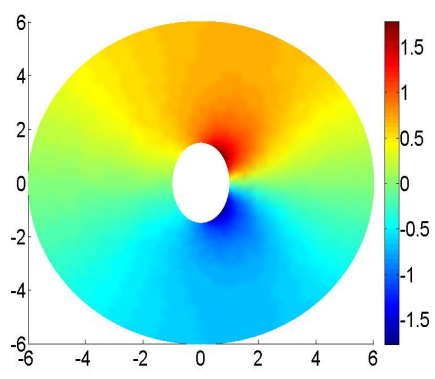

(b)

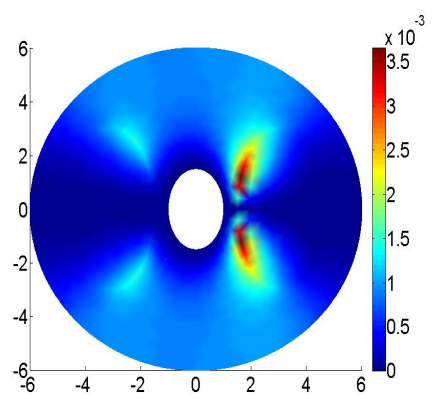

(e)

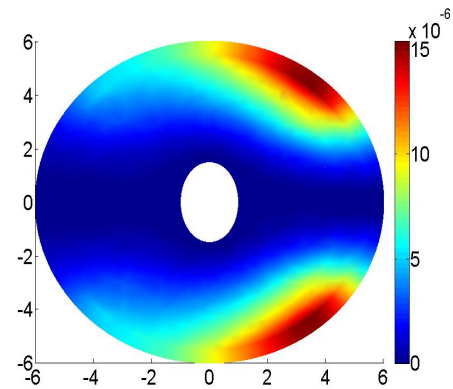

(c)

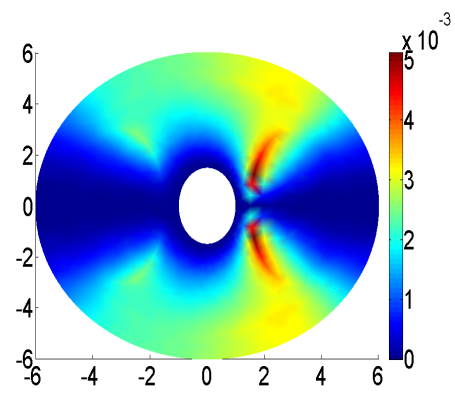

(f)

Figure 2: Mean value of the SFE (a). Exact deterministic solution (b). Standard deviation of the SFE solution for $\pm 50 \%$ of fat (respectively, muscle, lungs and torso cavity) conductivity (c) (respectively, (d), (e) and (f)).

\subsection{Sensitivity of the inverse solution to the conductivity uncertainties}

First, we consider the case where there is no uncertainties. Then we study the uncertainties effect of the conductivity of the fat (respectively muscle lung and torso cavity) on the solution of the inverse problem. In order to do that, we suppose that the conductivities of all organs are known (deterministic) except the conductivity of the fat (respectively muscle lung and torso cavity ) which follows a uniform law, and where we gradually increase the uncertainty from zero to $\pm 50 \%$ of the true conductivity value. We solve the stochastic inverse problem following the algorithm described in the previous section. We measure the effect of the uncertainties using relative error (RE) and the correlation coefficient (CC). In table 3, we show the RE and $\mathrm{CC}$ between the ground truth and the mean value of the stochastic optimal control solution. We used different level of uncertainties: $0 \%, \pm 3 \%, \pm 10 \%, \pm 20 \%, \pm 30 \%$ and $\pm 50 \%$. We find that the relative error of the inverse solution has been barely affected by the uncertainties of the fat and muscle conductivity even for high uncertainty levels. In fact, the RE (respectively, CC ) is 0.1202 (respectively, $0.9933)$ when there is no uncertainties. Introducing $\pm 50 \%$ of uncertainties in the fat conductivity gives a RE (respectively, CC) equal to 0.1249 (respectively, 0.9923). By the contrary the effect of the lung conductivity uncertainties is high: The RE increase from 0.1202 when we don't consider the uncertainties to 0.2932 when we introduce $\pm 50 \%$ of uncertainties on the lung conductivity. 


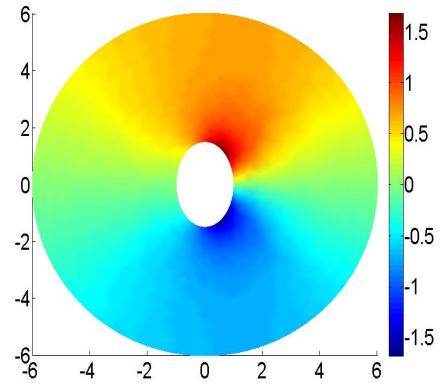

(a)

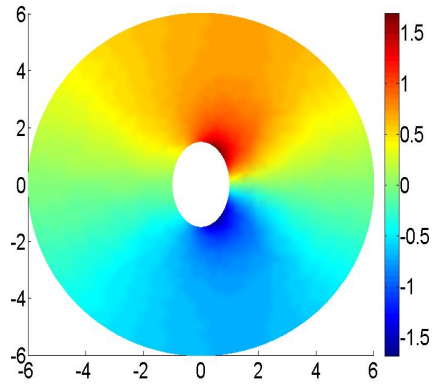

(b)

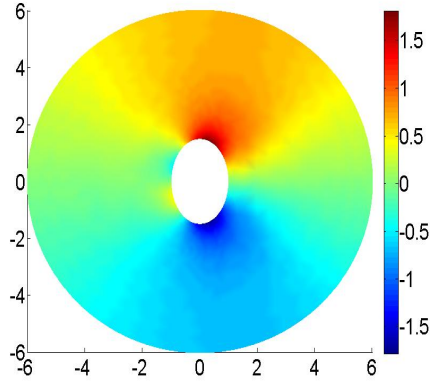

(c)

Figure 3: Mean value of the SFE solution with respect to null uncertainty in all organs model (a). Mean value of the SFE solution for $\pm 50 \%$ from the reference fat ( respectively, lungs) conductivity panel (b) (respectively, panel (c)).

The effect of the uncertainty on the correlation coefficient could also be qualitatively seen in Figure 3 , where the pattern of the mean value of the stochastic inverse solution looks the same in Figure 3 (a) (no uncertainties) and (b) ( $\pm 50 \%$ of uncertainties on the fat conductivity) and different in Figure 3 (c) ( $\pm 50 \%$ of uncertainties on lungs conductivity). Similarly the effect of uncertainties on the relative error could qualitatively seen in Figure 4. As shown in Table 3, the error does not change to much from no uncertainties (Figure 4 (a)) to $\pm 50 \%$ of fat conductivity uncertainty (Figure 4 (b)). Whereas the error is high for $\pm 50 \%$ of lungs conductivity uncertainty (Figure 4 (c)). The propagation of uncertainties from the conductivities to the inverse problem solution is reflected in the deviation of the stochastic inverse solution from the ground truth presented in Figure 5. We remark that the error is concentrated in the heart boundary $\Gamma_{\mathrm{i}}$, it reaches 0.8 for $\pm 50 \%$ of lungs conductivity uncertainty and 0.25 for $\pm 50 \%$ of fat conductivity uncertainty.

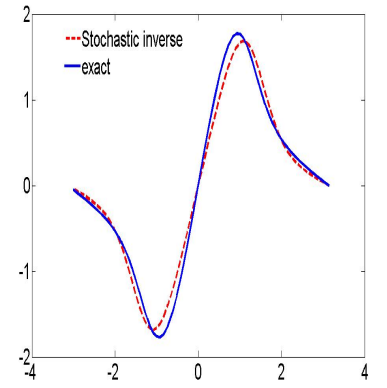

(a)

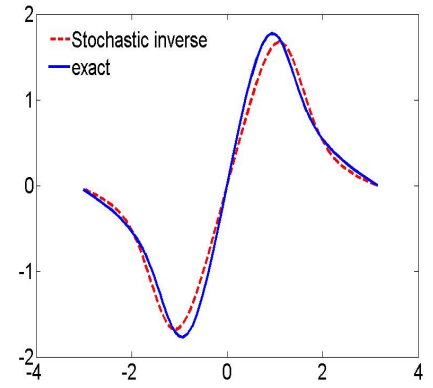

(b)

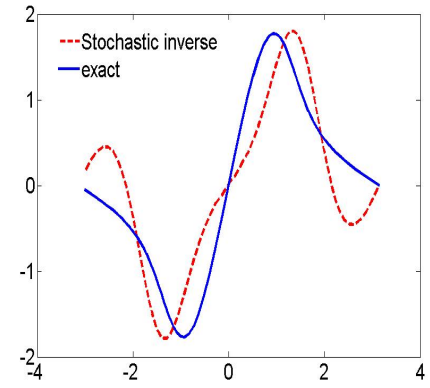

(c)

Figure 4: The effects of adding uncertainty regions of conductivity on the inverse solution in the epicardial boundary (missing data boundary ): Exact solution (blue continuous line). Stochastic inverse solution (red dashed line). No uncertainty (panel a ), $\pm 50 \%$ from the reference fat conductivity (panel b) and $\pm 50 \%$ from the reference lungs conductivity (panel c). X-axis polar coordinate angle from $-\pi$ to $\pi$. Y-axis value of the electrical potential on the boundary $\Gamma_{\mathrm{i}}$ corresponding to the polar coordinatite. 


\begin{tabular}{|c|c|c|c|c|c|c|c|}
\hline & conductivity uncertainties & $0 \%$ & $\pm 3 \%$ & $\pm 10 \%$ & $\pm 20 \%$ & $\pm 30 \%$ & $\pm 50 \%$ \\
\hline \multirow{3}{*}{ relative error } & fat & 0.1202 & 0.1202 & 0.1248 & 0.1233 & 0.1243 & 0.1249 \\
\cline { 2 - 7 } & muscle & 0.1202 & 0.1204 & 0.1277 & 0.1279 & 0.1272 & 0.1283 \\
\cline { 2 - 7 } & lungs & 0.1202 & 0.1286 & 0.1439 & 0.2108 & 0.2651 & 0.2932 \\
\cline { 2 - 7 } & cavity & 0.1202 & 0.1355 & 0.1597 & 0.2208 & 0.2813 & 0.4887 \\
\hline \multirow{3}{*}{ Corr coeff } & fat & 0.9933 & 0.9931 & 0.9928 & 0.9926 & 0.9926 & 0.9923 \\
\cline { 2 - 7 } & muscle & 0.9933 & 0.9930 & 0.9924 & 0.9924 & 0.9923 & 0.9921 \\
\cline { 2 - 7 } & lungs & 0.9933 & 0.9922 & 0.9899 & 0.9767 & 0.9654 & 0.9117 \\
\cline { 2 - 7 } & cavity & 0.9933 & 0.9909 & 0.9878 & 0.9799 & 0.9640 & 0.8802 \\
\hline
\end{tabular}

Table 3: Relative error and correlation coefficient of the stochastic inverse solution for different levels of uncertainty on the fat, muscle, lungs and cavity conductivities.

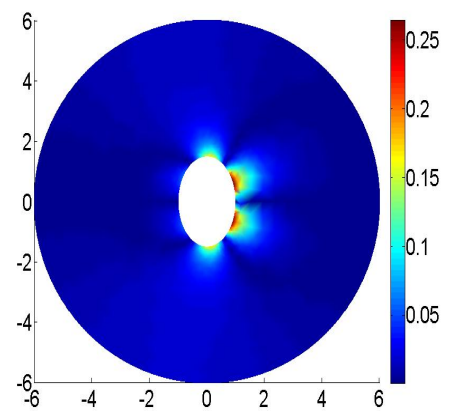

(a)

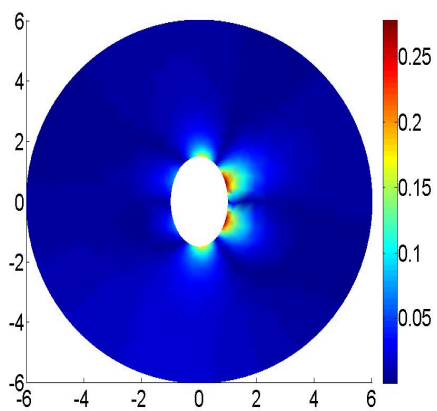

(b)

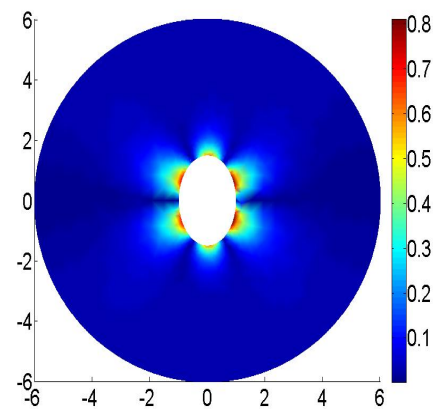

(c)

Figure 5: Panel (a) (respectively, panel (b) and (c)):Deviation between the SFE solution and exact solution with respect to null uncertainty in all organs model (respectively, $\pm 50 \%$ from the reference fat and lungs conductivity)

\subsection{Sensitivity of the inverse solution to the distance between the complete and incomplete boundaries}

In this paragraph, we study the influence of the distance between the complete boundary $\Gamma_{c}$ and the incomplete boundary $\Gamma_{i}$. First fix the minor and major radius of the internal ellipsoid boundary to the value 1.0 and $1.5 \mathrm{~cm}$. Then we gradually increase the radius of the circular external boundary from $3 \mathrm{~cm}$ to $4.5,8 \mathrm{~cm}$, and $9.5 \mathrm{~cm}$.

Figure 6 shows that the deviation between the SFE solution and exact solution with respect to null uncertainty, and for $\pm 50 \%$ uncertainties on fat, and lung. In other hand we observe that the variation of the radius of $\Gamma_{c}$ affect seriously the solution with null uncertainty, and we can also remark that the difference between the results obtained with null uncertainty and those with $\pm 50 \%$ fat uncertainties become clearly different with respect to the results obtained in the previous sections of the forward and the inverse problem. In Table 4, we show the obtained results for the fat and the lungs, we observe that the $\mathrm{CC}$ and RE deteriorate when we increase the distance between $\Gamma_{c}$ and $\Gamma_{i}$. For instance, the $\mathrm{RE}$ when considering $\pm 50 \%$ of fat conductivity uncertainty is $\mathrm{RE}=0.0098$ (respectively, $\mathrm{RE}=0.2$ ) when the external radius $=3 \mathrm{~cm}$ (respectively, $9.5 \mathrm{~cm}$ ). The effect is more significant for the lung conductivity uncertainty: $\mathrm{RE}=0.1548, \mathrm{CC}=0.988$, when the external radius $=3 \mathrm{~cm}$ and $\mathrm{RE}=0.5031, \mathrm{CC}=0.8731$ for an external radius of $9.5 \mathrm{~cm}$. 


\section{No uncertainty}

$3 \mathrm{~cm}$

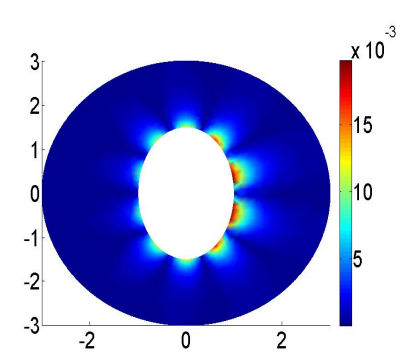

$4.5 \mathrm{~cm}$
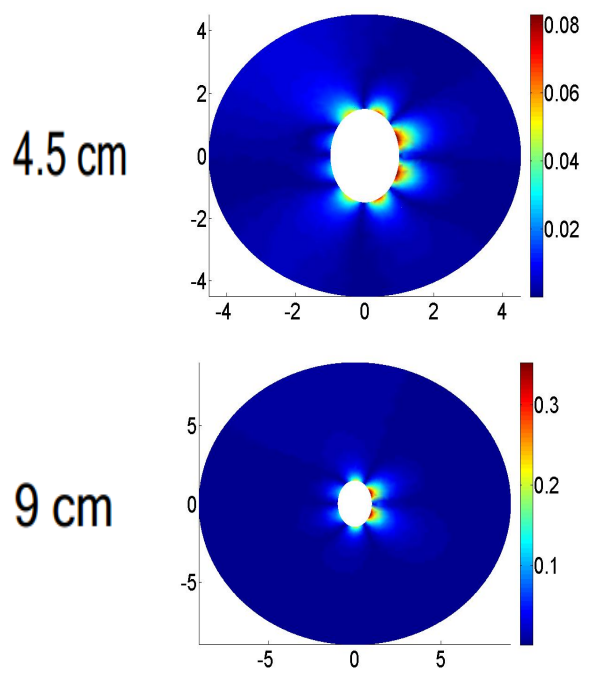

$\pm 50 \%$ uncertainty on fat
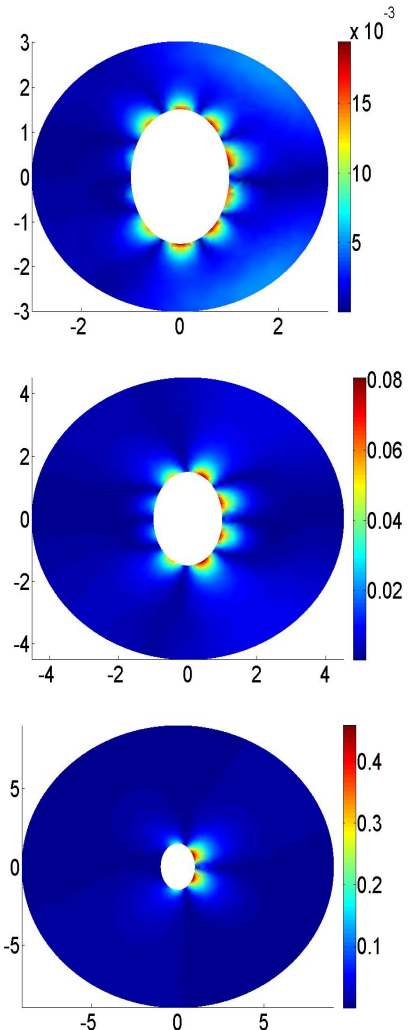

$\pm 50 \%$ uncertainty on lung
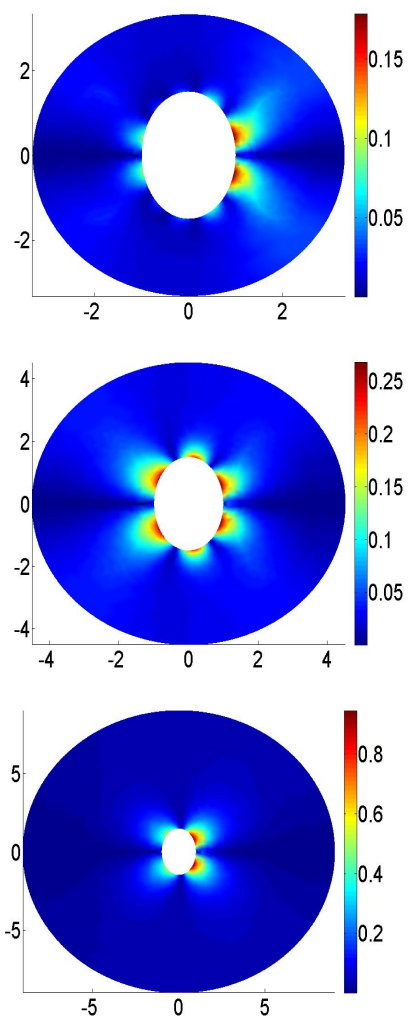

Figure 6: Left (respectively, middle, right) Deviation between the SFE solution and exact solution with respect to null uncertainty, (respectively $\pm 50 \%$ from the reference fat and lungs conductivity). from top to bottom results obtained for the external circular boundary Radius $=3 \mathrm{~cm}, 4.5 \mathrm{~cm}$ and $9.5 \mathrm{~cm}$.

\begin{tabular}{|l|c|c|c|c|c|c|c|c|}
\hline & \multicolumn{2}{|c|}{ radius=3 } & \multicolumn{2}{c|}{ radius $=4.5$} & \multicolumn{2}{c|}{ radius=8 } & \multicolumn{2}{c|}{ radius=9.5 } \\
\cline { 2 - 9 } & fat & lung & fat & lung & fat & lung & fat & lung \\
\hline Corr coeff & 1.000 & 0.9888 & 0.9991 & 0.9866 & 0.9842 & 0.9061 & 0.9823 & 0.8731 \\
\hline relative error & 0.0098 & 0.1548 & 0.04 & 0.1667 & 0.1826 & 0.2873 & 0.1999 & 0.5031 \\
\hline
\end{tabular}

Table 4: Relative error and correlation coefficient of the stochastic inverse solution for $\pm 50 \%$ from the reference fat and lungs conductivity for $2 \mathrm{D}$ torso geometry with different radius

\section{Electrocardiography imaging inverse problem}

In this section, we test the robustness of the methodology developed in the previous section for solving the inverse problem in electrocardiography imaging using a real life geometry.

\subsection{Anatomical model}

We segment a 2D slice of an MRI image of a 56 years old man. The MRI measures the diffusion of water molecules in biological tissues, which is useful to distinguish different regions in the torso domain. The segmentation of the slice shown in Figure 7 (left) is performed manually. We distinguish four organs: the heart surface, lungs, muscles (cavity) and fat. After the segmentation, we construct a $2 \mathrm{D}$ mesh of the torso cross section in which we identify the organs as shown in Figure 7 (right). In this representation, 

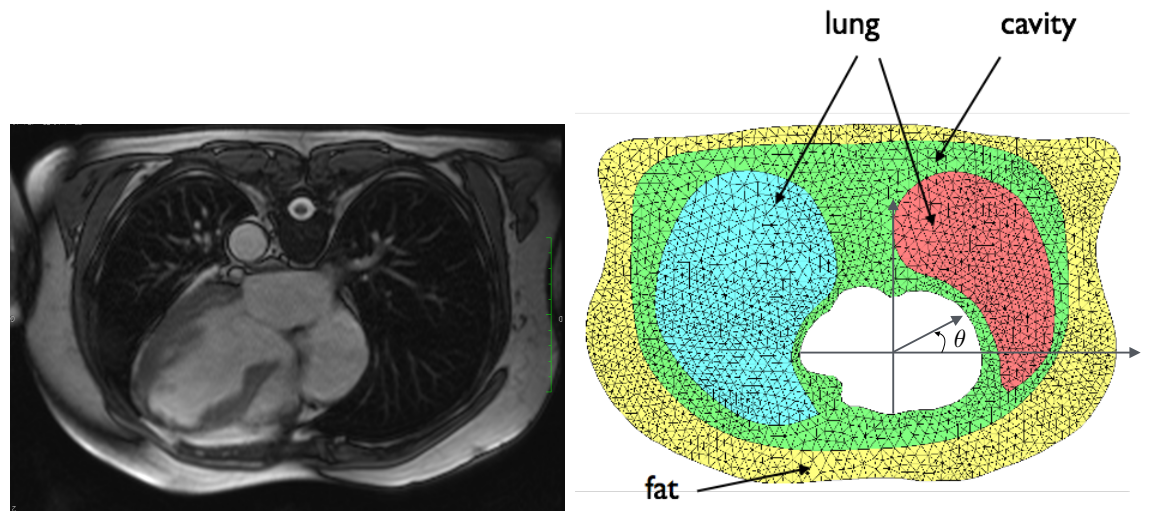

Figure 7: MRI 2D slice of the torso (left), 2D computational mesh of the torso geometry showing the different regions of the torso considered in this study: fat, lungs and torso cavity, (right). The angle $\theta$ is the second polar coordinate.

we consider that all the cavity region is occupied by the muscles. The $2 \mathrm{D}$ mesh contains 2395 vertices and 4540 elements.

\subsection{Numerical results}

In order to assess the effect of torso conductivity heterogeneities on the ECGI inverse solution, we generate synthetical data using the bidomain model in the heart domain. Since we suppose that the torso is a passive conductor, the electrical potential in the torso is governed by the Laplace equation and the conductivity depends on the domain as described. The heart is fully coupled to the torso ensuring the continuity of the electrical potential and current $[6,12]$. We extract the body surface potential at a given time step, it represents the boundary value on $f$ on the complete boundary $\Gamma_{c}$. Then, we solve the inverse problem following the algorithm described in section 3.2 In Figure 8 (left), we show the exact (or forward problem) solution.

\begin{tabular}{ccc}
$-4.8918 \mathrm{E}+00$ & \multicolumn{2}{c}{$4.5509 \mathrm{E}+00$} \\
$-9.6132 \mathrm{E}+00$ & 1 & 1 \\
$-1.7044 \mathrm{E}-01$ & $9.2723 \mathrm{E}+00$
\end{tabular}

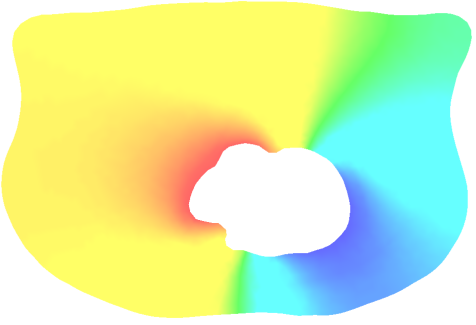

(a)

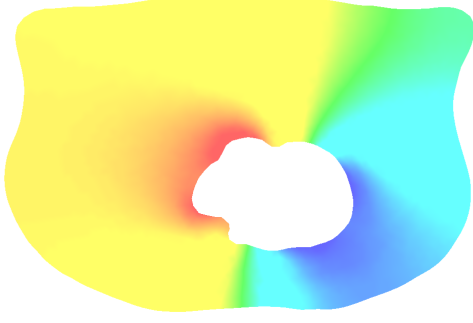

(b)

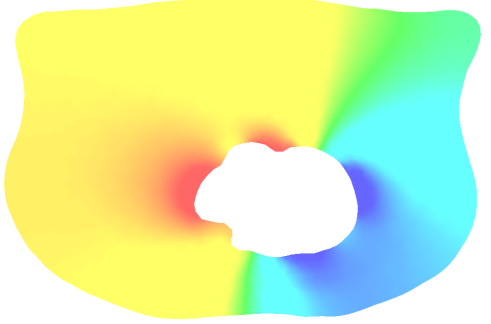

(c)

Figure 8: Effect of the conductivity uncertainties on the torso potential inverse solution: The figure (a) shows the exact forward solution. The figure (b) shows the inverse solution when no uncertainty is introduced. The Figure (c) shows the inverse solution when introducing $\pm 50 \%$ of lung conductivity uncertainty. 
The inverse solution in the deterministic case (meaning that no uncertainty is considered) is given in Figure 8 (middle). As shown in the analytical geometry case, when we consider the uncertainty on the lung conductivity, the inverse solution is affected: In Figure 8 (c), we show the distribution of the mean value of the ECGI inverse solution when assuming $\pm 50 \%$ of uncertainty on the lung conductivity. In Figure 9, we plot the electrical potential on the epicardial boundary $\Gamma_{i}$ versus the second polar coordinate $\theta$ (as represented in Figure 7). We compare the exact solution (blue continus line) to the inverse solution
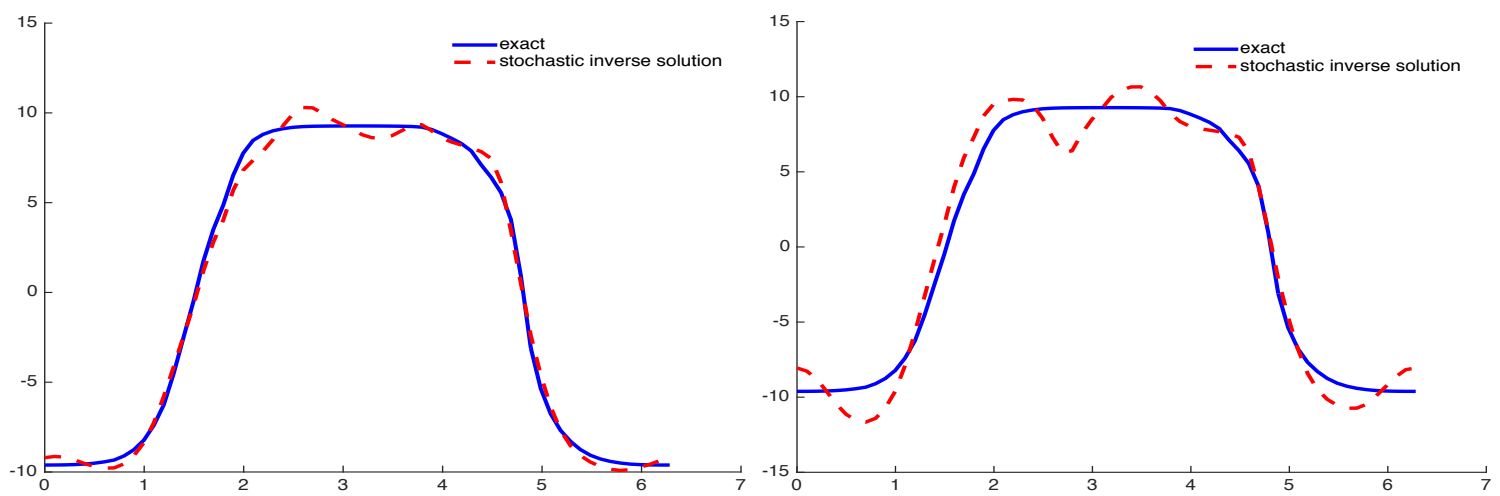

Figure 9: The effects of adding uncertainty of conductivity values on the inverse solution in the epicardial boundary (missing data boundary). Left (respectively, right): Epicardial potential when the uncertainty is equal to zero (respectively, $\pm 50 \%$ from the reference lungs conductivity). Exact solution (blue continuous line). Stochastic inverse solution (red dashed line). $\mathrm{X}$-axis denote the polar coordinate angle $\theta$ and vary from 0 to $2 \pi$. Y-axis value of the electrical potential on the boundary $\Gamma_{\mathrm{i}}$.

(red dashed line). The case where we don't consider uncertainty is given in Figure 9 (left), the relative error is $6 \%$. The case where we consider $\pm 50 \%$ of uncertainty on the lung conductivity is shown in Figure 9 (right), the relative error is $16 \%$. The standard deviation of the inverse solution in case of $\pm 50 \%$ of uncertainty on the lung conductivity is given in Figure 10. We remark that the standard deviation

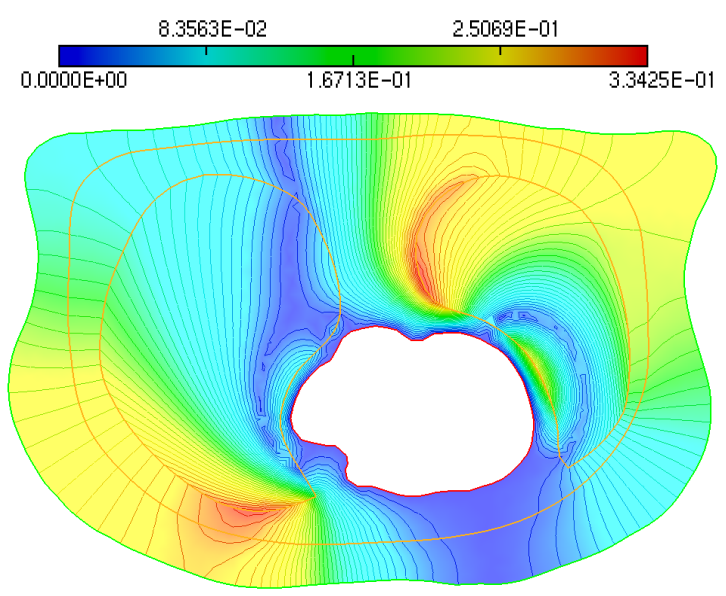

Figure 10: Standard deviation of the stochastic torso potential inverse solution when $\pm 50 \%$ of uncertainty is considered on the conductivity of the lungs 
magnitude is low compared to the mean value of the inverse solution. We also see that the of the standard deviation iso-values change direction when they cross the lung domain. This feature has been reported in [15]. We performed the same test for the fat and the cavity conductivities. In the cavity domain as represented in Figure 7, we consider the conductivity of the muscles. The inverse solution for $\pm 50 \%$ of uncertainty on the fat (respectively, muscle) conductivity is given in Figure 11 (left, (respectively right)). The distribution of the standard deviation of the stochastic inverse solution is provided in Figure 12 (left,
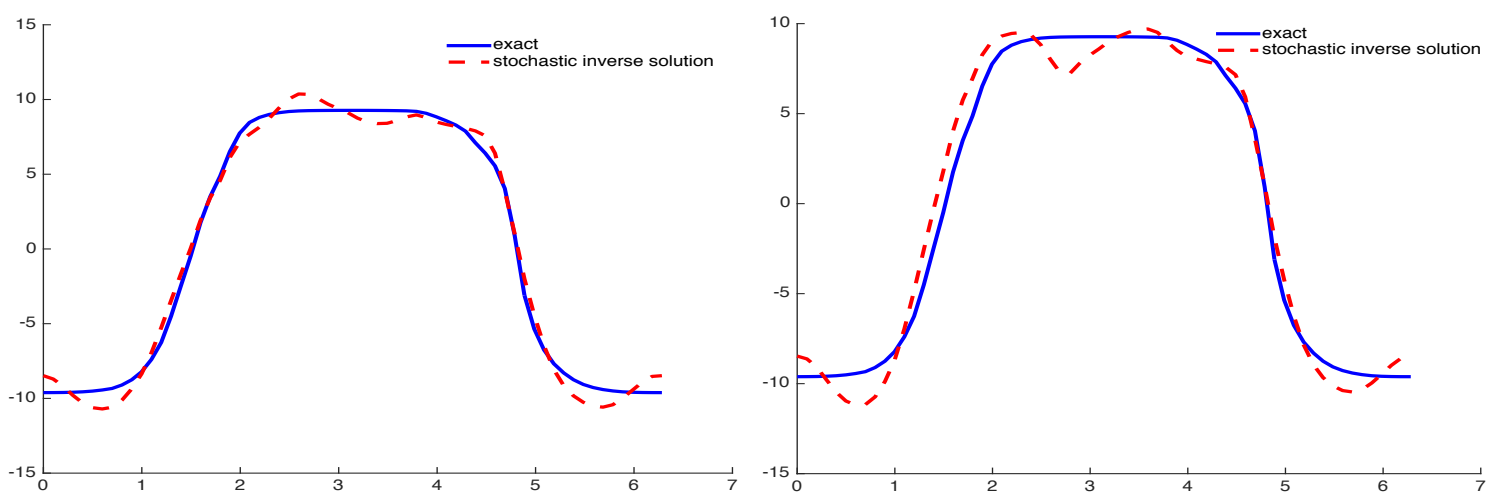

Figure 11: The effects of adding uncertainty of conductivity values on the inverse solution in the epicardial boundary (missing data boundary). Left (respectively, right): Epicardial potential when the uncertainty on the fat (respectively, muscle) conductivity is equal to $\pm 50 \%$. Exact solution (blue continuous line). Stochastic inverse solution (red dashed line). X-axis denote the polar coordinate angle $\theta$ and vary from 0 to $2 \pi$. Y-axis value of the electrical potential on the boundary $\Gamma_{\mathrm{i}}$.

(respectively right)). The relative error is $9.5 \%$ for the fat case and $13.6 \%$ for the muscle case.

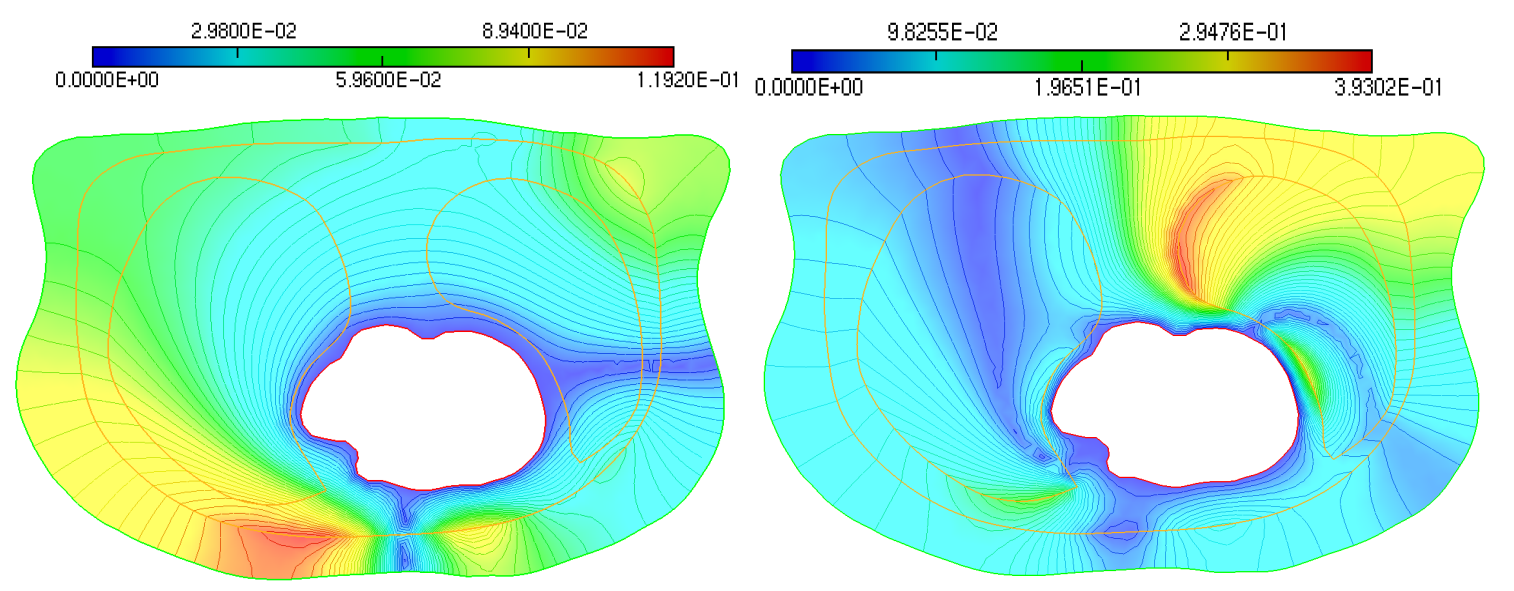

Figure 12: Left (respectively, right): Standard deviation of the stochastic torso potential inverse solution when $\pm 50 \%$ of uncertainty is considered on the conductivity of the fat (respectively muscle). 


\section{Discussion}

Solving the inverse problem in electrocardiography imaging based on a combination of an optimal control approach and the SFEM allowed us to quantify the effect of the torso organs conductivity uncertainties on the ECGI inverse solution. We highlight the fact that the stochastic approach provides a complete spatial distribution of the conductivity uncertainty effects on the forward and the inverse problem. This allows to obtain a mean value and a standard deviation of the solution at all points of the heart surface. Whereas, deterministic approaches only provide global measure of the error between the exact and the inverse solutions. Our results show that increasing the level of the fat conductivity uncertainty from zero to $\pm 50 \%$ of its original value does not alter too much the quality of the reconstructed potential. This is in line with the results presented in [15] for the forward problem when introducing $\pm 50 \%$ uncertainties in the fat conductivity. On the contrary, the results that we obtained for the uncertainties on the lungs conductivity show an important effect on the ECGI solution. In fact the relative error is about $16 \%$ when introducing $\pm 50 \%$ of uncertainty and the $\mathrm{CC}$ is significantly altered. This result is different from the results presented in [15] for the forward solution with $\pm 50 \%$ uncertainties in the lungs conductivity where the standard deviation does not exceed $\pm 3 \%$ of the mean value. We also have shown that the isolines of the standard deviation change directions when they cross the organ on which we have uncertainties. This is expected and in line with the results obtained in [15]. The standard deviation of the inverse solution reflects the same features as the Std of the forward SFEM solution.

\section{Conclusions}

In this work we presented a novel approach to solve the inverse ECG problem using a stochastic optimal control formulation. This formulation allowed us to study sensitivity to parameters values in data completion inverse problem and that could have application in a wide range of bioelectric and biomedical inverse problems resolution. We used a stochastic finite element method in order to take into account the variability of the conductivity values in the ECGI inverse problem formulated in a stochastic optimal control problem. We used a conjugate gradient method to solve this problem where the gradient of the cost function was computed using an adjoint method. We have described the different steps of the algorithm used to solve this stochastic inverse problem. The numerical simulation that we conducted in the $2 \mathrm{D}$ analytical geometry and in the $2 \mathrm{D}$ cross section of a real torso showed that there is an important sensitivity of the solution to the lungs and the skeletal muscle conductivity uncertainties, whereas the uncertainties on the fat conductivity did not affect too much alter the inverse solution. One of the major challenges that we would like to address in future works is the implementation in 3D of the methodology that we presented in this paper and see if the same results would be obtained in the $3 \mathrm{D}$ case. This task is challenging because of the intrusively of the SFEM in the standard finite element libraries.

Acknowledgements. We would like to thank the LIRIMA Laboratory which financially supported the EPICARD associate team to perform this study. This work was also supported by the Agence Nationale de la Recherche (Grant IHU LIRYC ANR-10-IAHU-04)

\section{References}

[1] R. Aboulaich, A. B. Abda, M. Kallel. Missing boundary data reconstruction via an approximate optimal control. Inverse Problems and Imaging, 2 (4) (2008), 411-426.

[2] S. Andrieux, T.N. Baranger, A.B. Abda. Solving cauchy problems by minimizing an energy-like functional. Inverse problems, 22 (1) (2006), 115-133.

[3] I. Babuska, R. Tempone, G.E. Zouraris. Solving elliptic boundary value problems with uncertain coefficients by the finite element method: the stochastic formulation. Comput. Methods Appl. Mech. Engrg, 194 (2005), $1251-1294$.

[4] I. Babuska, R. Tempone, G.E. Zouraris. Galerkin finite element approximations of stochastic elliptic partial differential equations. SIAM Journal on Numerical Analysis, 42 (2) (2005), 800-825.

[5] G. Blatman, B. Sudret. An adaptive algorithm to build up sparse polynomial chaos expansions for stochastic finite element analysis. Probabilistic Engineering Mechanics, (2010), 183-197. 
[6] M. Boulakia, S. Cazeau, M.A. Fernández, J.F. Gerbeau, N. Zemzemi. Mathematical modeling of electrocardiograms: a numerical study. Annals of biomedical engineering, 38 (3) (2010), 1071-1097.

[7] P. Chen, A. Quarteroni, G. Rozza. Stochastic optimal robin boundary control problems of advection-dominated elliptic equations. SIAM Journal on Numerical Analysis, 51 (6) (2013), 3163-3185.

[8] O. Doessel, Y. Jiang, W.H. Schulze. Localization of the origin of premature beats using anintegral method. International Journal of Bioelectromagnetism, 13 (2011), 178-183.

[9] F.A. Duck. Physical Properties of Tissue: A Comprehensive Reference Book. London, England: Academic, Harcourt Brace Jovanovich, 1990.

[10] M. Eiermann, O.G. Ernst, E. Ullmann. Computational aspects of the stochastic finite element method. 10 (1) (2007), $3-15$.

[11] T.J.C. Faes, D.M.H. Van, D.J.C Munck, R.M. Heethaar. The electric resistivity of human tissues (100 hz-10 mhz): a meta-analysis of review studies. Physiological measurement, 20 (4) (1999), 1-11.

[12] M.A. Fernández, N. Zemzemi. Decoupled time-marching schemes in computational cardiac electrophysiology and ecg numerical simulation. Mathematical biosciences, 226 (1) (2010), 58-75.

[13] K.R. Foster, H.P. Schwan. Dielectric properties of tissues and biological materials: a critical review. Critical reviews in biomedical engineering, 17 (1) (1988), 25-104.

[14] S. Gabriel, R. Lau, C. Gabriel. The dielectric properties of biological tissues: Ii. measurements in the frequency range $10 \mathrm{hz}$ to $20 \mathrm{ghz}$. Physics in medicine and biology, 41 (11) (1996), 22-51.

[15] S.E. Geneser, R.M. Kirby, R.S. MacLeod. Application of stochastic finite element methods to study the sensitivity of ecg forward modeling to organ conductivity. Biomedical Engineering, IEEE Transactions on, 55 (1) (2008), 31-40.

[16] R. Ghanem, P. Spanos. Stochastic Finite Elements:a Spectral Approach. Springer-Verlag, 1991.

[17] S. Ghosh, Y Rudy. Application of l1-norm regularization to epicardial potential solution of the inverse electrocardiography problem. Annals of Biomedical Engineering, 37 (5) (2009), 902-912.

[18] J. Hadamard. Lectures on Cauchy's problem in linear partial differential equations. Yale University Press, New Haven, 1923.

[19] P.C. Hansen, D.P. O'Leary. The use of the l-curve in the regularization of discrete ill-posed problems. SIAM Journal on Scientific Computing, 14 (6) (1993), 1487-1503.

[20] L. S Hou, J. Lee, H. Manouzi. Finite element approximations of stochastic optimal control problems constrained by stochastic elliptic pde. Journal of Mathematical Analysis and Applications, 384 (1) (2011), 87-103.

[21] O.P. Le Maître, M.T. Reagan, H.N. Najm, R.G. Ghanem, O.M. Knio. A stochastic projection method for fluid flow: Ii. random process. Journal of computational Physics, 181 (1) (2002), 9-44.

[22] V.A. Oosterom, G.J. Huiskamp. The effect of torso inhomogeneities on body surface potentials quantified using "'tailored"' geometry. Journal of electrocardiology, 22 (1) (1989), 53-72.

[23] A. Rouatbi. Complétion de données via des méthodes de type controle. Master's thesis, LAMSIN, E.N.I.T. Tunis, 2009.

[24] A.J. Shah, H.S Lim, S. Yamashita, S. Zellerhoff, B. Berte, S. Mahida, D. Hooks, N. Aljefairi, N. Derval, A. Denis, and al. Non invasive ecg mapping to guide catheter ablation. JAFIB: Journal of Atrial Fibrillation, 7(3), 2014.

[25] F.M. Weber, D.U. Keller, S. Bauer, G. Seemann, C. Lorenz, O. Dossel. Predicting tissue conductivity influences on body surface potentialsan efficient approach based on principal component analysis. Biomedical Engineering IEEE Transactions, 58 (2) (2011), 256-273.

[26] S. Wiener. The homogeneous chaos. Am. J. Math. vol. 60, (1998), 897-936.

[27] D. Xiu, G.E.Karniadakis. The wiener-askey polynomial chaos for stochastic differential equations. Journal on Scientific Computing, 24 (2002), 619-644.

[28] E.V. Zakharov, A.V. Kalinin. Algorithms and numerical analysis of dc fields in a piecewise-homogeneous medium by the boundary integral equation method. Computational Mathematics and Modeling, 20 (3), (2009), 247-257. 